\title{
Using the Global Obstetric Fistula Electronic Registry to Digitalize Patient Records in Kathmandu Model Hospital
}

\author{
Hema Kumari Pradhan, ${ }^{1}$ Ganesh Dangal, ${ }^{1}$ Aruna Karki, ${ }^{1}$ Ranjana Shrestha, ${ }^{1}$ Kabin Bhattachan, ${ }^{1}$ \\ Rekha Poudel, ${ }^{1}$ Nishma Bajracharya, ${ }^{1}$ Kenusha Devi Tiwari, ${ }^{1}$ Seth Cochran, ${ }^{2}$ Bryony Michaelson ${ }^{2}$ \\ ${ }^{1}$ Department of Obstetrics and Gynaecology, Kathmandu Model Hospital; \\ ${ }^{2}$ Operation Fistula, London, UK.
}

DOI: http://dx.doi.org/10.3126/njog.v13i2.21709

\begin{abstract}
Aims: The aim of the study was to collect and digitalize data of patients who underwent fistula repair at Kathmandu Model Hospital. Once data were collected, a Tableau dashboard was built to visualize the collected data and to share key insights.

Methods: Operation Fistula built a digital version of Kathmandu Model Hospital's Surgery Log, using a mobile data collection tool (CommCare). Researchers were trained on the data collection tool and provided with an electronic tablet device which hosted the application. Kathmandu Model Hospital then proceeded to input patient records, meanwhile providing Operation Fistula with key feedback to continue improving this process.
\end{abstract}

Results: The results of this process have been threefold: facilitation of data collection, creation of an accurate database and provision of clear insights with access to data visualization dashboards.

Conclusions: This study has shown the benefits of digitizing historical patient records using Operation Fistula's Global Obstetric Fistula Electronic Registry (GOFER) platform. By creating an online database, this has the potential to be used for future academic research.

Keywords: fistula repair, GOFER, operation fistula.

\section{INTRODUCTION}

Obstetric fistula is a disease of the developing world, resulting from prolonged obstructed labour, hysterectomy and other gynaecological surgeries. Women suffer for years as treatment services are rare. Even when treatment is available, often women are completely unaware that fistula can be repaired or they lack resources for the cost of operative care. ${ }^{1,2}$ Obstetric fistula is the epitome of gender inequality. It only occurs where healthcare systems profoundly fail women and girls. The fistula leaves them abandoned by their families and stigmatized by their communities. ${ }^{3}$ Like most issues linked to gender inequality, obstetric fistula is missing critical data. This is primarily because data is difficult to collect and process. True prevalence is not known as patients live with fistula for years. Reliable data on prevalence and burden of disease is sparce. ${ }^{4-6}$ The commonly quoted prevalence estimate is 2 million cases worldwide and 50,000100.000 cases each year. ${ }^{7,8}$ A recent survey of 298 health officials from 64 lower middle income class revealed that they think their data is low in quality yet high in influence. Furthermore, 20\% reported a

\section{CORRESPONDENCE}

Dr Hema Kumari Pradhan

Kathmandu Model Hospital

Email: drhemapradhan@hotmail.com time lag from data collection to data publication of more than 3 years. Without visibility into their efforts and progress, service providers cannot improve their performance, and governments cannot make policies to provide better quality of care. ${ }^{3}$

\section{USE OF GLOBAL OBSTETRIC FISTULA ELECTRONIC REGISTRY (GOFER)}

The Global Obstetric Fistula Electronic Registry (GOFER) is founded by operations engineer $\mathrm{Mr}$. Seth Cochran, whose team at Operation Fistula is dedicated to fighting the global injustice of women suffering from obstetric fistula. The shared vision is to use GOFER as a platform to unite and improve the fistula sector, to introduce visibility into quality care, to improve outcomes of obstetric fistula surgery and to expand the impact of performance-based funding. GOFER makes the task of entering data more efficient and reveals greater insight into patient tracking and clinical performance. It is designed to document and improve every interaction between each fistula patient and her care team. The platform powers maps and dashboards that are accessible to every stakeholder in the continuum of care, who can use them to monitor their individual performance in real-time. The data also powers predictive analytics machines that help find new cases and recognize 
performance improvement opportunities within the clinical setting. This process will ultimately lead to more concrete knowledge of limits and barriers which impede the efficiency of fistula care, and will help guide stakeholders within the fistula sector to be more informed in their decision making. ${ }^{3}$

By using these technological and analytical capacities, Operation Fistula's aim, with the input and guidance from Kathmandu Model Hospital is to develop surveillance systems that map and monitor obstetric fistula. This effort eventually helps to identify new cases of fistula wherever gender inequality is most severe. It also allows other organizations to use and share the data on obstetric fistula to find other marginalized populations who have also been affected by this systemic failure.

\section{DIGITALIZATION OF RECORDS OF FISTULA SURGERY BY USING} COMMCARE, ALTERYXAND TABLEAU

This project was centred around the digitalization of records of obstetric fistula surgery in Kathmandu Model Hospital. The data were collected from the records of the patients operated for fistula surgery. The system consisted of several technological tools that were deployed for various needs. Researchers were trained on the data collection tool and provided with a tablet which hosted the application. For data collection, the Operation Fistula team built a customized digital form based on the physical patient data collection sheet available from Kathmandu Model Hospital, which was hosted on the CommCare application. The researchers provided Operation Fistula with key feedback to continue improving this process. The collected data were uploaded to several cloud-based databases (Figure 1). Alteryx software was used to prepare and connect the data from the database to the Tableau platform. As the project progresses, the team aims to include Exasol, a business intelligence tool used for database management, to automatically merge these datasets along with geographic and population data from the national government and multilaterals. This creates the data warehouse that powers our performance management system and analysis/visualization engine. The primary visualization tool used was Tableau, software used for data visualization. The Operation Fistula Analytics team used Tableau to create intuitive maps and dashboards (Figures 2 and 3) that were populated as performance data was entered into the data collection tools. These dashboards generated further questions that we investigated. This study included records of 263 patients operated for fistula in Kathmandu Model Hospital from 2014 until 2018. These dashboards generated further questions that we will be investigating further.

\section{VARIABLES USED IN COMIMCARE}

The digital form includes two broad fields: patient particulars and surgery log. The patient particulars included name, ID, region, zone, district and address. Surgery log included date of surgery, new or repeat patient, current age, years living with fistula, parity, number of living children, type of incontinence, presumed cause of fistula, previous surgery, surgical diagnosis \#1, surgical diagnosis \#2, surgical procedure $\# 1$, surgical procedure \#2, GOH classification, difficulty of surgery (simple, intermediate, difficult), outcome of surgery (dry, fistula closed, incontinent, fistula not closed), surgeon-in-charge, days in hospital, number of catheter days, date of discharge, complications, comments. The examples of dashboard are shown in Figure 2 and 3.

$\begin{array}{ll}* \text { Researcher } & \dagger \text { Mobile with CommCare app } \\ \text { †CommCare cloud } & \S \text { Analyst }\end{array}$

qStakeholder

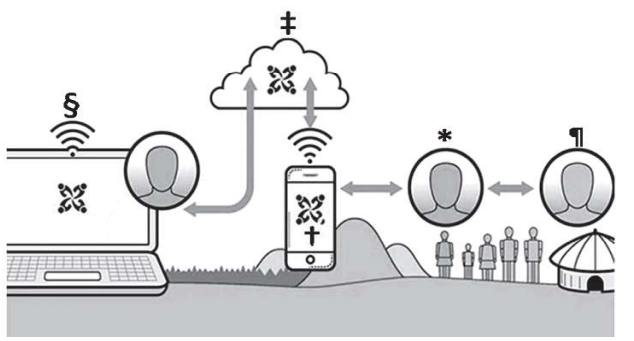

Figure 1. Basic version of an information flow diagram.

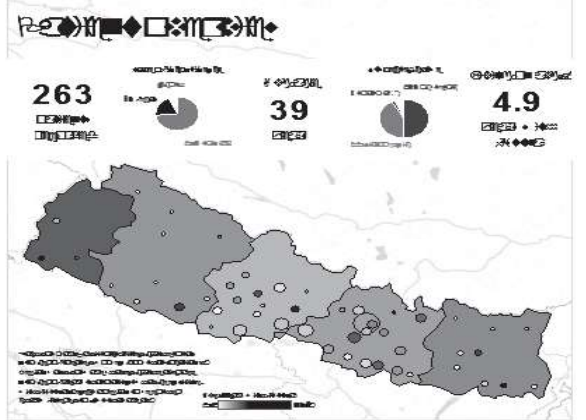

Figure 2. Patient overview dashboard, created with Tableau software. 


\section{Surgical outcomes}

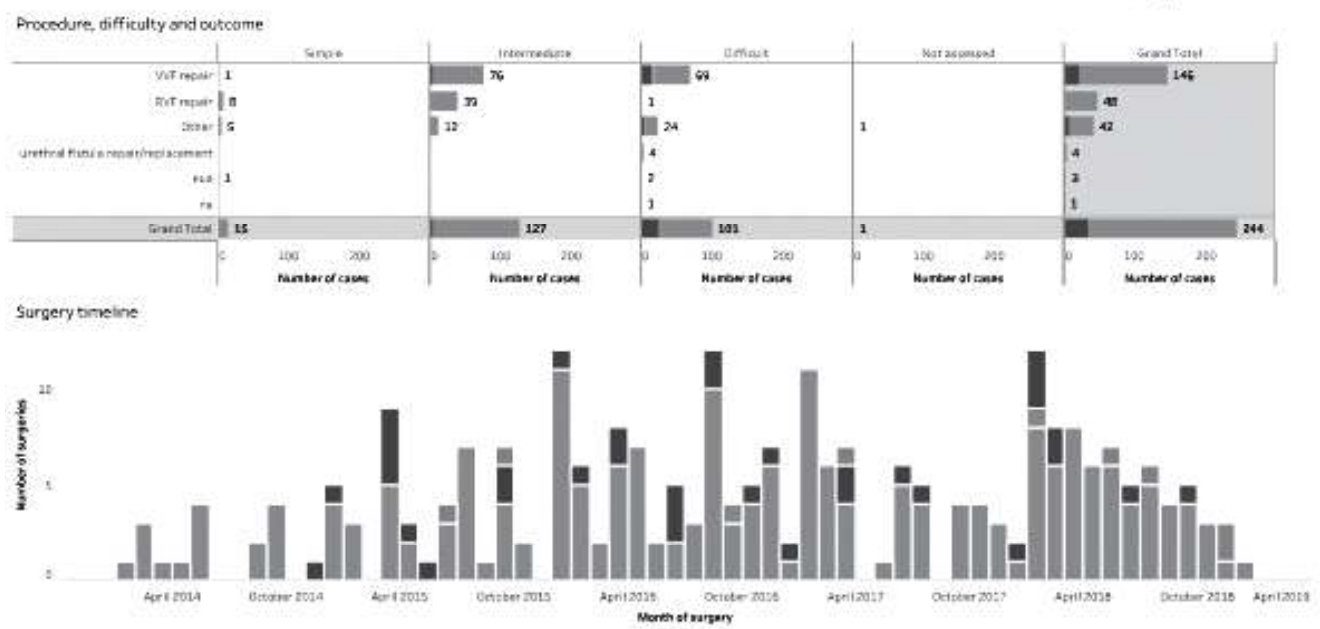

Figure 3. Surgical Outcomes and timeline visualization -Kathmandu Model Hospital dashboard, created with Tableau software.

There are several advantages of electronic data collection. It avoids mistakes before they happen by warning the end user, asks the right questions based on in-built form logic, gives multiple question formats for better quality data response from the end user, lets us know how the work is progressing, eliminates data entry errors and experimentally tests survey design. ${ }^{9}$ It provides a case management system which allows the end user to track the patient's care. CommCare tracks data fast, works offline and uploads data when internet is available, empowers end users, stores ultimate data on secured cloud server, incorporates performance feedback for regular progress update. 10 While initially collecting data in CommCare used by GOFER, we realized the need of improvement in few areas, like data for duration of patient suffering is difficult to express in terms of days or months, repeat surgery on the same patient is recorded as new surgery $\log$, no record of attempted surgery of fistula at other institute, .The team at Kathmandu Model Hospital is in communication with Operation Fistula about these issues and are working together to customise their app further to suit these needs.

\section{CONCLUSIONS}

This study has shown the benefits of digitizing patient records using GOFER. By creating an online database, this has the potential to be used for future academic research.

\section{ACKNOWLEDGEMENT}

Thanks to Operation Fistula for logistics and technical support.

\section{REFERENCES}

1. Lewis G, de Bernis L, editors. Obstetric fistula: guiding principles for clinical management and programme development. Geneva: World Health Organization; 2006.

2. Miller S, Lister F, Webster M, Cowan B. Obstetric fistula: a preventable tragedy. J Midwifery Women's Health. 2005;50(4):286-94.

3. Operation fistula pilot program report. 2012-2015.

4. Wall LL. Obstetric vesico-vaginal fistula as an international public-health problem. Lancet. 2006;368:1201-9.

5. Zheng AX, Anderson FW. Obstetric fistula in low-income countries. Int J Gynaecol Obstet. 2009;104:85-9.

6. Stanton C, Holtz SA, Ahmed S. Challenges in measuring obstetric fistula. Int J Gynaecol Obstet. 2007;99(Suppl 1):S4-9.
7. Cottingham J, Royston E. Obstetric Fistula: A Review of Available Information. Geneva, Switzerland: World Health Organization; 1991.

8. Lewis G, de Bernis I, editors. Obstetric Fistula. Guiding Principles for Clinical Management and Programme Development. Geneva, Switzerland: World Health Organization; 2006. [www.who.int/reproductive-health/docs/ obstetric_fistula/obstetric_fistula.pdf]. Accessed 26 October 2011.

9. Electronic vs paper based data collection: reviewing the debate, Development Impact Guest Blogger, 25/5/2016. www. blogs.worldbank.orghttp://www.dimagi.com 